archives

of thermodynamics

Vol. 37(2016), No. 1, 47-72

DOI: $10.1515 /$ aoter-2016-0004

\title{
Utilization of the horizontal ground heat exchanger in the heating and cooling system of a residential building
}

\author{
MAEGORZATA HANUSZKIEWICZ-DRAPAŁA ${ }^{1}$ \\ TOMASZ BURY
}

Silesian University of Technology, Institute of Thermal Technology, Konarskiego 22, 44-100 Gliwice, Poland

\begin{abstract}
This paper presents the results of thermodynamic analyses of a system using a horizontal ground heat exchanger to cool a residential building in summer and heat it in the autumn-winter period. The main heating device is a vapour compression heat pump with the ground as the lower heat source. The aim of the analyses is to examine the impact of heat supply to the ground in the summer period, when the building is cooled, on the operation of the heating system equipped with a heat pump in the next heating season, including electricity consumption. The processes occurring in cooling and heating systems have an unsteady nature. The main results of the calculations are among others the time-dependent values of heat fluxes extracted from or transferred to the ground heat exchanger, the fluxes of heat generated by the heat pump and supplied to the heated building by an additional heat source, the parameters in characteristic points of the systems, the temperature distributions in the ground and the driving electricity consumption in the period under analysis. The paper presents results of analysis of cumulative primary energy consumption of the analyzed systems and cumulative emissions of harmful substances.
\end{abstract}

Keywords: Heat pump; Ground heat exchanger; Heating system, Numerical modelling

\footnotetext{
${ }^{1}$ Corresponding Author. E-mail: malgorzata.hanuszkiewicz-drapala@polsl.pl
} 


\section{Nomenclature}

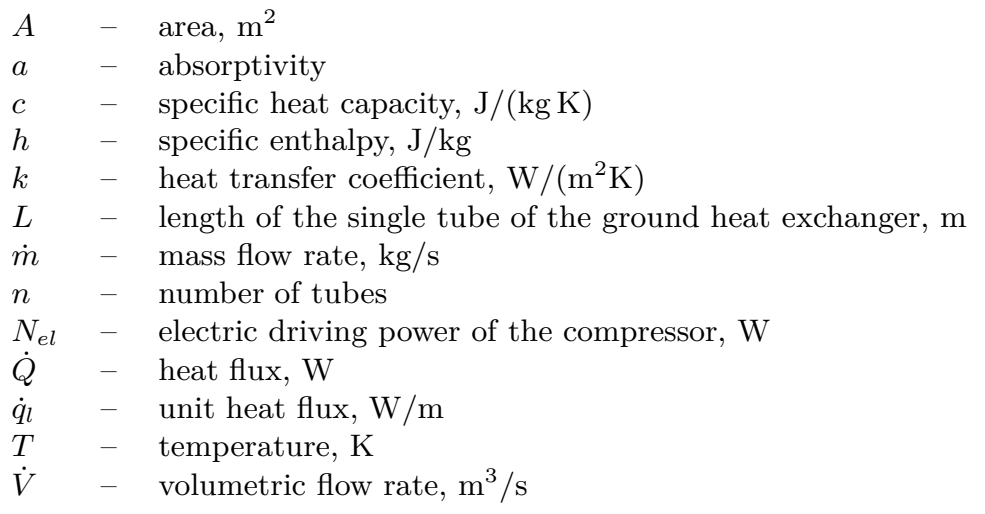

\section{Greek symbols}

$\alpha-\quad$ convection heat transfer coefficient, $\mathrm{W} /\left(\mathrm{m}^{2} \mathrm{~K}\right)$

$\varepsilon \quad-$ emissivity

$\lambda-$ coefficient of thermal conductivity, $\mathrm{W} /(\mathrm{m} \mathrm{K})$

$\rho \quad-\quad$ density, $\mathrm{kg} / \mathrm{m}^{3}$

$\tau \quad-$ time, $\mathrm{s}$

$\Delta \tau \quad-\quad$ time step, $\mathrm{s}$

$\eta_{i s} \quad-\quad$ internal efficiency of the compressor

\section{Subscripts}

$\begin{array}{lll}a & - & \text { air } \\ a v & - & \text { average values } \\ c & - & \text { condenser, condensation } \\ c s & - & \text { cooled space } \\ c s e & - & \text { heat exchangers in the cooled space } \\ e & - & \text { evaporator, evaporation } \\ g & - & \text { ground } \\ g l & - & \text { heat carrier in the ground heat exchanger } \\ H E & - & \text { heat exchanger in the cooling system } \\ h s & - & \text { heated space } \\ h s e & - & \text { heat exchangers in the heated space } \\ r & - & \text { refrigerant } \\ w & - & \text { water }\end{array}$

\section{Introduction}

In the times of environmental protection against the harmful effects of conventional generation of heat and electricity, the use of renewable energy sources is becoming more and more popular. One of such energy sources is the rock mass with its petrothermal or hydrothermal resources. Local high- 
temperature hydrothermal energy resources in the world make it possible to generate electricity in the organic Rankine cycle systems, for example. Generally, however, the rock mass is characterized by a relatively low temperature, which limits its potential for power generation. Its application in Polish conditions is practically limited to installations generating heat for heating purposes. Such installations with heat pumps may be supplied with geothermal fluids or with agent being the heat carrier in the process of heat extraction from the rock mass. At present, the number of heat-pump based heating installations reaches several dozen millions. Another method of the use of the rock mass in terms of energy is cooling the buildings in summer. One of the ways to lower the temperature inside the building is to supply it with ventilating air cooled in an appropriate (tube or gravel) heat exchanger. In order to overcome the flow resistance, a fan has to be used, which involves higher energy consumption. Using this solution, it is also possible to preheat ventilating air in the autumn-winter period. A slightly different solution is to use in summer a heating system with a vapour compression heat pump, operating reversibly as a cooling device. In such a situation, it is obvious that electricity has to be supplied to drive the compressor. A simultaneous effect of this solution is the supply of heat to the ground, cooled after the heating season, which affects the heating system operation in the next winter period.

This work presents the results of thermodynamic analyses of the heating and cooling system of a small residential building. The demand for heat (cool) varies in time. The main heat source in the heating system is a vapour compression heat pump extracting heat from the ground through a heat carrier circulating in the tubes of a horizontal heat exchanger. The heating system is additionally equipped with a conventional peak heat source. The heat is transferred to the building through water that flows in heat exchangers located in the heated space. In summer the building is cooled using the heat exchangers mentioned above and an extra water-tointermediate fluid heat exchanger. In this case, the heat carrier flows in the tubes of the ground heat exchanger and transfers heat to the ground. The processes occurring in cooling and heating systems have an unsteady nature. The aim of the analyses was to check the functionality of this solution due to the possibility of cooling the building in summer. The impact of supplying the ground with heat obtained in the cooling process on the operation of the heat pump unit in the next heating season was also analyzed. The obtained results were compared with the results of the case 
when the ground thermal regeneration in the spring-summer period is the effect of natural processes only. The calculations were performed using an in-house computer program using the ANSYS Fluent software package [1]. One of the calculation results is the electricity consumption in the systems under consideration. Based on that, the cumulative energy consumption of the heating and cooling systems using a horizontal ground heat exchanger and cumulative emissions of harmful substances was determined.

Heat pump systems and ground heat exchangers (horizontal and vertical) have been investigated for years. The works are based on experiments and simulations [2-8]. Some of them concern the influence of heat pump systems on natural environment and energy consumption. Results of analysis of geothermal heat plant using low-temperature water are presented in $[9]$.

\section{Mathematical models of the building's heating and cooling systems}

\subsection{Heating system for the building}

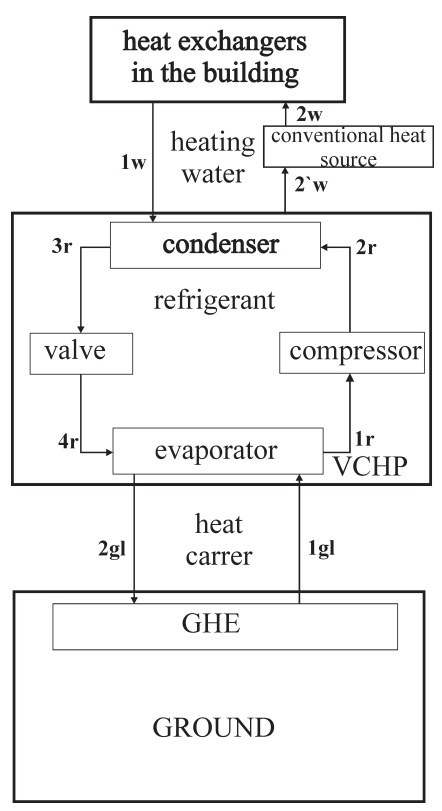

Figure 1: Diagram of the heating system. 
The buildings heating system (Fig. 1) is composed of a vapor compression heat pump unit (VCHP), a horizontal ground heat exchanger (GHE) and heat exchangers - heaters in the heated space [10-12]. A heat carrier - an aqueous solution of propylene glycol - circulates in the GHE composed of parallel tubes. This medium extracts heat from the rock mass and transfers it to the heat pump evaporator. The effect is the refrigerant vaporization. It was assumed, that the VCHP works according to the Linde cycle with irreversible adiabatic compression. The condenser is cooled with water the heating medium in the heat exchangers in the building. During the heating season, the demand for heat varies. It depends on factors such as the building orientation to the sides of the world or instantaneous values of the environmental parameters characteristic of a given location.

The developed model of the building $[10,12]$ assumes a heat exchange with the surroundings through radiation and convection at varied values of solar radiation heat fluxes and values of ambient temperatures, taking account of the unsteady processes of heat transfer through the building walls and in the building floor - ground system. These problems are solved using the ANSYS Fluent program which is called within the in-house computer code as a procedure for determination of the fabric temperature distributions and unit heat fluxes transferred from the air in the building to the inside surfaces of the walls, the roof and the floor. Calculations were performed at a constant time step, $\Delta \tau$, for repeatable, two-dimensional elements: parts of the building walls, roof and floor situated on the ground. Sample elements of the wall and the subsystem floor - ground are presented in Fig. 2.

Values of total heat transferred from the heated space to: inner surfaces of the walls, $\sum_{j} \dot{Q}_{w a l l} j \Delta \tau$ (where $j$ is the direction of the world), the roof, $\dot{Q}_{\text {roof }} \Delta \tau$, and the floor, $\dot{Q}_{\text {floor }} \Delta \tau$, in the time step $\Delta \tau$ were calculated on the basis of unit heat fluxes mentioned above. It was assumed constant air temperature inside the heated space. Energy balance equation of the heated space

$$
\begin{aligned}
& \dot{Q}_{g} \Delta \tau+\sum_{j} \dot{Q}_{w i n j} \Delta \tau+\dot{Q}_{g i n} \Delta \tau= \\
& =\sum_{j} \dot{Q}_{\text {wall } j} \Delta \tau+\dot{Q}_{\text {roof }} \Delta \tau+\dot{Q}_{\text {floor }} \Delta \tau+\dot{Q}_{\text {wout }} \Delta \tau+\dot{Q}_{v} \Delta \tau+\dot{Q}_{h b} \Delta \tau
\end{aligned}
$$

allows to calculate demand for heat in elementary time step $\dot{Q}_{g} \Delta \tau[10,12]$. Apart from heat losses mentioned above, the energy balance (1) takes into 


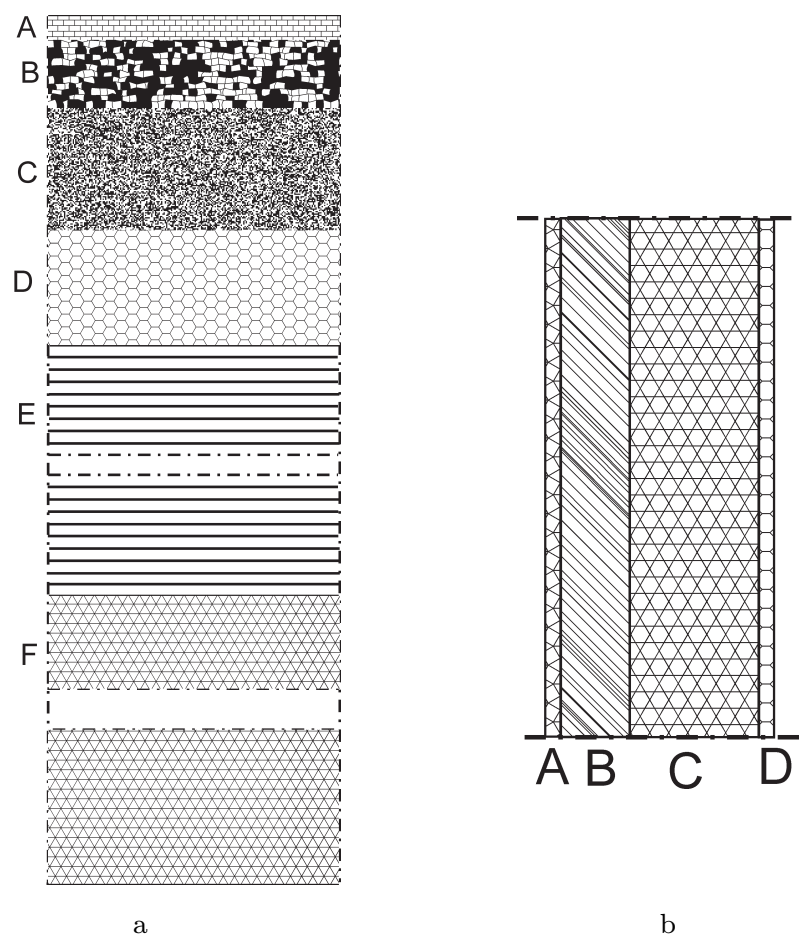

Figure 2: Repeatable elements: a) building's floor - ground (A - terracotta, B - jointless floor, $\mathrm{C}$ - thermal insulation, D - lean concrete, E - sand, F - ground); b) building's wall (A, D - plasters, $\mathrm{B}$ - thermal insulation, $\mathrm{C}$ - wall material).

account heat losses, $\dot{Q}_{w o u t} \Delta \tau$, and gains, $\sum_{j} \dot{Q}_{w i n j} \Delta \tau$, through the windows, internal heat gains, $\dot{Q}_{g i n} \Delta \tau$, ventilation heat losses, $\dot{Q}_{v} \Delta \tau$, or losses related to heat bridges, $\dot{Q}_{h b} \Delta \tau$.

The numerical program makes it possible to determine the total demand for heat at any time using Eq. (1) in consecutive time steps. The demand for cool in summer was calculated in a similar way. Mathematical model of VCHP was presented in [10-12]. The processes in the vapour compression heat pump systems proceed very slowly. Therefore, the operation of individual devices of the heat pump and heat exchangers in the building is described using energy balance equations for instantaneous steady states in each time step. The operation of heat exchangers in the analyzed system is also described using the Peclet equations, taking account of the variability heat transfer coefficients. The fundamental equations describing the operation of the VCHP and the heat exchangers in the heated space in each time 
step are presented in Appendix (Eqs. (1a)-(12a)) [10-12]. The set of equations mentioned above is supplemented with the formulae for determination of calorific parameters of the intermediate carrier and refrigerant and with compressor operating characteristic. The set of nonlinear equations obtained in this manner was solved iteratively in each time step [10-12]. The scheme of the GHE is shown in Fig. 3a. Mathematical model of the GHE is based on the assumption resulting from a practical aspect concerning the small increment in the intermediate medium temperature in such exchangers $(2-4 \mathrm{~K})[10-11]$. Due to that, it is assumed that the average unit heat

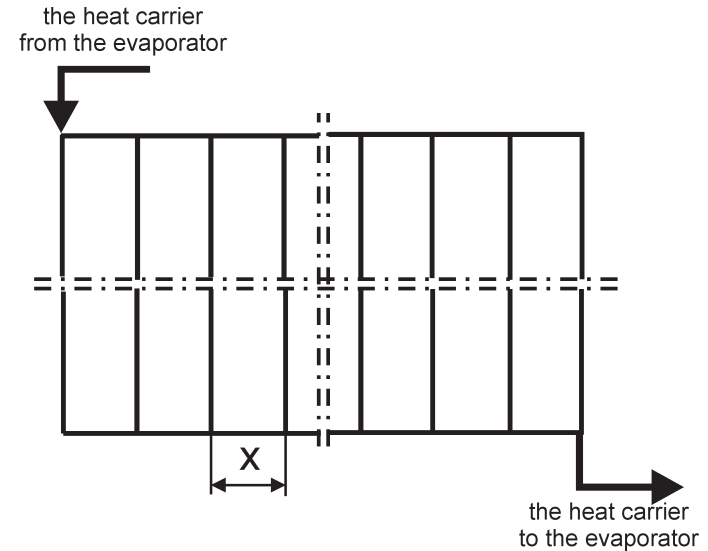

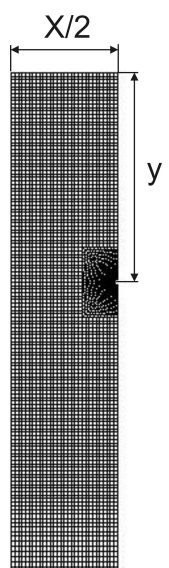

Figure 3: Horizontal ground heat exchanger: a) diagram; b) part of the calculation area: $\mathrm{x}$ - distance between tubes, $\mathrm{y}$ - depth of the tube location.

flux extracted from the ground and the unit heat flux calculated for an instantaneous average value of temperature of the heat carrier in the tube of the GHE are equal. This assumption makes it possible to analyze thermal processes taking place in the area of the GHE in a two-dimensional system $[10,11]$. The calculations were performed for the reproducible part of the system: GHE - ground (Fig. 3b). This system is delimited by the ground surface (top), the plane parallel to the ground located at the depth of $20 \mathrm{~m}$ (bottom) and two vertical adiabatic planes, one of which includes the exchanger tube axis and the other is located halfway the distance between neighbouring tubes. A mixed convection-radiation heat transfer occurs between the ground surface and the surroundings characterized by changeable parameters, temperatures and solar radiation heat fluxes [10]. The model takes into account the phase change of the moisture contained in the ground 
through assumption of the increased value of specific heat capacity of the ground in elements where the phase change occurs [10]. In the analyzed cases the infiltration of ground water was ignored. The calculation results for each time step include the temperature distribution in the analyzed part of the system GHE - ground, the unit heat flux extracted from the ground and the outlet temperature of the heat carrier, $T_{1 g l}$, for the given inlet temperature $T_{2 g l}$. The method of finding the above-mentioned heat flux in each time step has an iterative character. The calculations were carried out based on the following

$$
\begin{gathered}
T_{g l a v}=\frac{T_{1 g l}+T_{2 g l}}{2}, \\
\dot{Q}_{e}=\dot{q}_{l} L n, \\
T_{1 g l}=T_{2 g l}+\frac{\dot{Q}_{e}}{\dot{V}_{g l} c_{g l} \rho_{g l}}, \\
\dot{q}_{l}=\dot{q}\left(T_{g l a v}\right), \\
\frac{\partial^{2} T_{g}}{\partial x^{2}}+\frac{\partial^{2} T_{g}}{\partial y^{2}}=\frac{\rho_{g} c_{g}}{\lambda_{g}} \frac{\partial T_{g}}{\partial \tau} .
\end{gathered}
$$

using the commercial software (academic version of ANSYS Fluent) to determine the temperature distribution in the ground and the unit heat flux, $\dot{q}_{l}$, extracted therefrom. Moreover, the model takes into account the variability of the volumetric flow rate of the heat carrier in the GHE $[10,12]$. The instantaneous value of the volumetric flow rate is found based on the circulation pump characteristics

$$
H_{p}=F_{p}\left(\dot{V}_{g l}\right)
$$

and variable hydraulic resistances of this medium flow [10]

$$
H_{r}=F_{r}\left(\dot{V}_{g l}, T_{\text {glav }}\right) .
$$

This is related to the changes in the medium properties caused by changes in its temperature. The result is the variability of the boundary condition on the outer surface of the tube - in each time step the values of the equivalent heat transfer coefficient and of the heat carrier average temperature are determined:

$$
k_{g l}=k\left(\dot{V}_{g l}, T_{g l a v}\right) .
$$


In the comprehensive model there was assumed a cyclic operation of the heating system. The heating season was divided into steady balance periods for which the heat demand was determined $[10,12]$. It was assumed that the VCHP supplies heat to the building from the beginning of each balance period, step by step, and continues until the heat demand is satisfied. It does not operate for the rest of the balance period. In each balance period the calculations were performed assuming a constant time step. When the VCHP does not operate, heat is not extracted from the rock mass - the tubes are regarded as insulated $\left(k_{g l}=0\right)$ and the process of partial thermal regeneration occurs in the ground. In each time step the temperature distribution in the ground was determined. If the heat pump thermal output is insufficient to supply the required amount of heat, the heating system is supported by an additional conventional heat source. The VCHP then operates in a continuous manner throughout the entire balance period, step by step.

The computational process based on the comprehensive model of the system has in each time step an iterative character due to the adopted assumptions and the relationships among the operation of the three subsystems, i.e., heat exchangers in the heated building, VCHP and GHE, at variable parameters of the environment $[10,12]$. Operation of the heat pump evaporator and the functioning of GHE are closely interrelated due to the heat carrier circulation. It is therefore necessary to find the value of this medium temperature both at the ground exchanger inlet and outlet, i.e., at the evaporator outlet and inlet. A similar interrelation takes place in the heating water circuit (the condenser of the VCHP - the heat exchangers in the heated building). This implies solving the problem iteratively, taking account of the unsteady process of the heat transfer in the GHE and varied demands for heat [10-12]. Simplified diagram of the calculations in the balance period is presented in Appendix (Fig. 21).

The developed computer program $[10,12]$ allows long-term thermodynamic analyses of the heating system operation, e.g., for subsequent heating seasons, taking account of the process of the rock mass natural thermal regeneration in the spring-summer period. The calculation results for the heating season are, for example, the time-dependent values of temperature of the heat carrier at GHE inlet and outlet, values of the heat carrier mass flow rate, temperatures of evaporation and condensation, heating water temperatures, values of the fluxes of heat extracted from the ground and supplied to the building by VCHP and by the additional heat source. 
Moreover, the following are found: the values of the driving electric power of the compressor and of the intermediate agent circulation pump, temperature distributions in the ground around the heat exchanger tubes in each time step of the analyzed period, time of the heat pump unit operation in each balance period. For the entire heating season the electricity consumption and the amount of energy supplied by the additional heat source are determined.

\subsection{Building's cooling system}

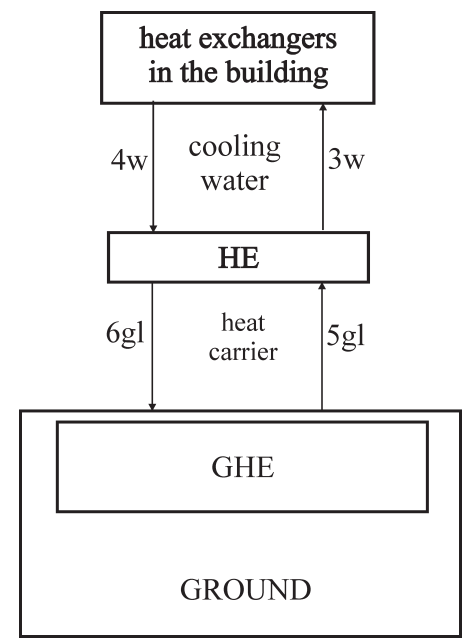

Figure 4: Diagram of the cooling system.

The diagram of the building's cooling system is presented in Fig. 4. Beside GHE and heat exchangers inside the building, the system includes an additional heat exchanger HE. The heat extracted from the air inside the building by the water circulating in the system of heat exchangers is transferred to the heat carrier, which - flowing through the system of the GHE tubes - gives the heat up to the ground. The mathematical model of this system includes the model of the subsystem: ground - GHE, which was described earlier. It is assumed that the temperature of the heat carrier at GHE inlet is equal to the temperature of this medium at the outlet of additional heat exchanger $\mathrm{HE}$, and temperature of the heat carrier at the outlet of e GHE tubes is the same as the temperature at the HE inlet. The operation of the HE and of the operation of the heat exchangers inside the 
building is described similarly to the model of the heating system, i.e., using energy balance equations and the Peclet equation, assuming steady operation in each time step (Appendix, Eqs.(13a)-(16a)). The variation of the volumetric flow rate of the heat carrier was taken into consideration in the same manner as for the model of the building heating system. The cooling process is cyclic. The summer season was divided into balance periods for which the demand for cool, determined using the mathematical model of the building mentioned above is known. The calculations for each balance period were made at a constant time step. The cooling system operates from the beginning of each balance period in subsequent time steps until the required amount of heat is extracted from the building. The system does not operate for the remaining part of the period. Until the period end, only the computational process of the unsteady heat conduction in the ground is realized. Due to the assumptions of the model, the computational cycle in each time step is iterative. The simplified diagram of the calculations in the balance period is presented in Appendix (Fig. 22). It was assumed that if the system efficiency is insufficient to extract the required amount of heat from the building, the heat extraction process has to be supported by an additional cooling device. Another assumption was that the system stops its operation if the temperature of the ground during the cooling system operation becomes too high to continue to extract heat from the building and dump it to the ground. After such a break in operation, an attempt was made to switch the system on again. The results of calculations are as follows: time-dependent changes in the heat flux extracted from the building, time of the system operation per 24 hours, characteristic temperatures of the mediums, temperature distributions in the ground in the vicinity of heat exchanger tubes. The consumption of electricity needed to drive the heat carrier pump in the GHE and the amount of heat extracted from the building cooled by the cooling system as well as the additional cooling device are determined for the selected cooling period.

\section{Basic data}

The simulations were carried out for a system with a GHE made of 10 parallel $30 \mathrm{~m}$ long tubes placed in the ground at the depth $y=1.5 \mathrm{~m}$ at a distance $x=0.75 \mathrm{~m}$ from each other. The outer diameter of the pipes is equal to $0.04 \mathrm{~m}$, and the thickness of the walls is equal to $0.0023 \mathrm{~m}$. The calculations assume that the ground consists of two parts [13]: 
- upper part, 0-10 m (unfrozen ground/frozen ground): product of density and specific heat capacity $-3740 \mathrm{~kJ} /\left(\mathrm{m}^{3} \mathrm{~K}\right) / 2600 \mathrm{~kJ} /\left(\mathrm{m}^{3} \mathrm{~K}\right)$, thermal conductivity $-1.5 \mathrm{~W} /(\mathrm{mK}) / 1.75 \mathrm{~W} /(\mathrm{m} \mathrm{K})$, latent heat of moist ground $80 \mathrm{~kJ} / \mathrm{kg}$;

- lower part, 10-100 m (unfrozen ground/frozen ground): product of density and specific heat capacity $-3220 \mathrm{~kJ} /\left(\mathrm{m}^{3} \mathrm{~K}\right) / 2330 \mathrm{~kJ} /\left(\mathrm{m}^{3} \mathrm{~K}\right)$, thermal conductivity $2.2 \mathrm{~W} /(\mathrm{m} \mathrm{K}) / 1.2 \mathrm{~W} /(\mathrm{m} \mathrm{K})$, latent heat of moist ground $55 \mathrm{~kJ} / \mathrm{kg}$.

At the upper boundary of the computational domain of GHE convective and radiative heat transfer was assumed $\left(\alpha=16 \mathrm{~W} /\left(\mathrm{m}^{2} \mathrm{~K}\right), a=0.8 \varepsilon=0.8\right)$, at the lower boundary - constant temperature $(282 \mathrm{~K})$, the vertical boundaries were assumed to be adiabatic [10,12]. The division into 3-hour balance periods was adopted in the calculations, assuming a constant time step (450 s). The initial temperature distribution in the computational domain (first heating season) is the result of heat transfer calculations in the ground without GHE [10]. The calculations comprise two years before the start of the system operation.

It was assumed that the mass flow rate of the heating water is $0.8 \mathrm{~kg} / \mathrm{s}$ and the value of the compressor volumetric capacity is constant at $0.00475 \mathrm{~m}^{3} / \mathrm{s}$. The electric driving power of the compressor is determined on the basis of its performance characteristic. The condenser has the following geometric parameters: number of pipes -42 , length of pipes $-1 \mathrm{~m}$, outside/inside pipe diameter $-0.012 \mathrm{~m} / 0.010 \mathrm{~m}$; and evaporator: number of pipes -42 , length of pipes $-0.7 \mathrm{~m}$, outside/inside pipe diameter - $0.012 \mathrm{~m} / 0.010 \mathrm{~m}$. The same are the heat exchanger (HE) parameters, i.e. number of pipes -42 , length of pipes $-0.7 \mathrm{~m}$, outside/inside pipe diameter $-0.012 \mathrm{~m} / 0.010 \mathrm{~m}$. In sample demonstration calculations the test fluid in the heat pump cycle was refrigerant R134a and water solution of propylene glycol was assumed as a heat carrier in GHE. Due to R134a thermal properties and the ozone depletion potential $\mathrm{ODP}=0$, this refrigerant is commonly used among others in vapor compression heat pumps (in practice at no too low temperature of heat carriers in ground heat exchangers), although its global warming potential, GWP $=1340$. Number of heat exchangers in heated space is equal to 8. During cooling process the number is equal to 12 . In the numerical calculation the product $k A$ (where stand for heat transfer coefficient and $A$ is the contact area) for these heat exchangers were determined on the basis of their thermal characteristic as a function of water temperatures. The values of demand for 
heat and demand for cool were determined for a contemporary residential building with a surface area of approximately $180 \mathrm{~m}^{2}$ and with the given structure [10]. The division into 3-hour balance periods was adopted in the calculations, assuming a constant temperature in the heated building of $22^{\circ} \mathrm{C}$, constant time step (450 s) and constant values of the convection heat transfer coefficients, the emissivity and the absorptivity [10]. Assumed in calculations solar radiation heat fluxes, temperatures of atmospheric air and temperatures of horizon dependent on time are characteristic for the city of Katowice [14]. Sample data are presented in Figs. 5 and 6. It was assumed that the heating season begins in mid-September and lasts for 230 days and that the building is cooled between June 20 and August 20.

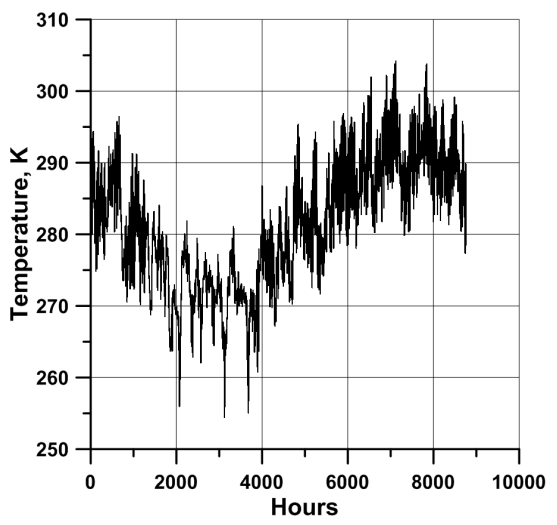

Figure 5: Variability of air temperature.

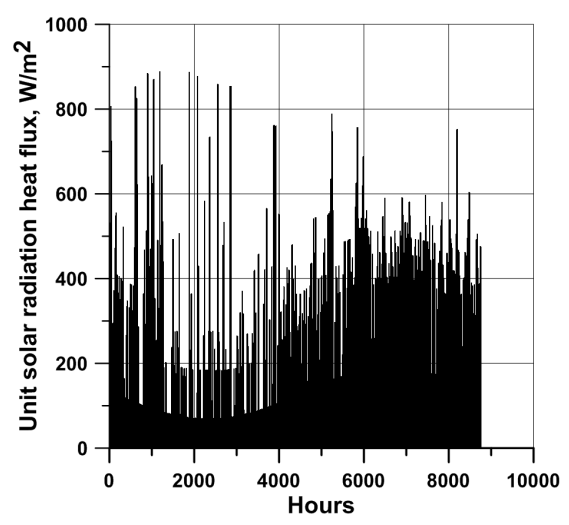

Figure 6: Variability of solar radiation heat flux (outh-west direction, $90^{\circ}$.

\section{Calculation results}

The developed mathematical models, described above, and the computer programs based on them enable to conduct thermodynamic analyses of the heating and cooling system. The calculations concern two heating seasons (season I and season II) and two variants. In the first variant, between the heating seasons - in the spring-summer period - natural thermal regeneration of the ground around the heat exchanger tubes occurs. In the second variant (denoted as $\mathrm{A}$ ), the heat extracted during the building cooling process is transferred to the ground, which affects the ground thermal state. Figure 7 presents the demand for heat, which is identical in each heating 
season, and Fig. 8 - the demand for cool in the spring-summer period. For analysed building the variabilities of demand for heat and demand for cool are strong dependent on ambient temperature changeability. It should be stressed that values of demand for heat and cool were calculated on the basis of statistical meteorological data from the long-term period [14].

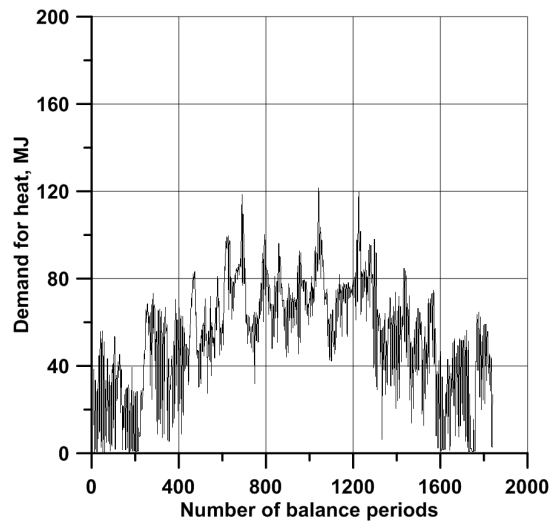

Figure 7: Demand for heat during the heating season.

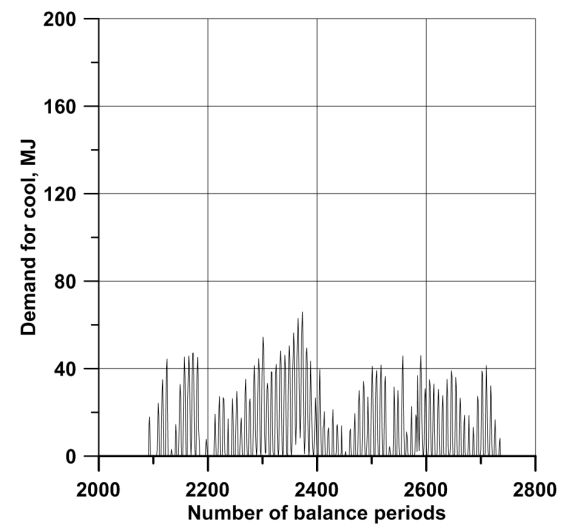

Figure 8: Demand for cool.
A

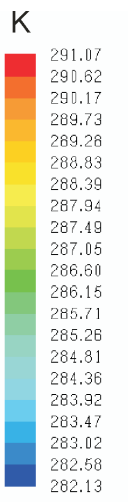

B

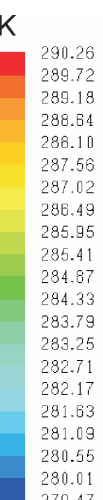

$\mathrm{K}$

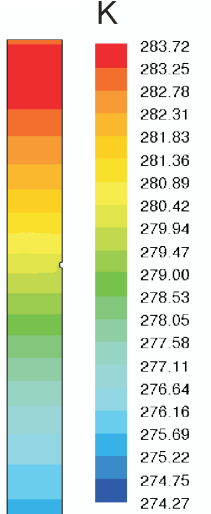

C

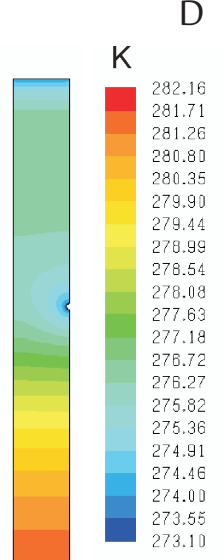

$\mathrm{E}$

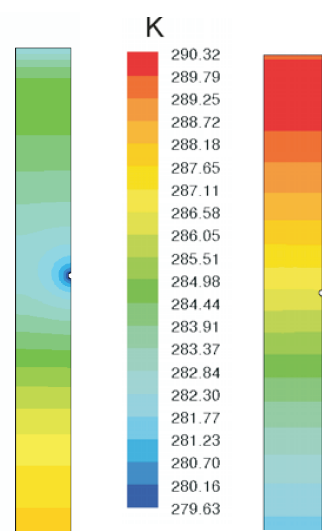

Figure 9: Temperature distributions in the ground: A) beginning of season I, B) beginning of season II, C) 70th day of season I, D) 70th day of season II, E) beginning of season IIA.

Selected results of the calculations concerning two-season analyses of the operation of the heating and cooling systems are presented in Figs. 9-20. 
Average values per 24 hours are calculated in relation to the time of operation of the systems per day and night.

\subsection{Calculation results of the heating system}

The thermal state of the ground around the GHE tubes varies during the operation of the heating system [10-12]. This results from the extraction of heat by the heat carrier flowing in the GHE tubes and from the heat exchange with the surroundings on the ground surface. It should be mentioned that the ground thermal state depends also on the frequency of operation of the additional heat source in the heating system. As proved by previous analyses $[10,11]$, the heat fluxes extracted from the ground are bigger in season I than in season II. This is the effect of the incomplete thermal regeneration of the ground in the spring-summer period, which, as mentioned above, has a natural character. In consequence, the temperature distributions in the vicinity of the GHE before the beginning of the first and second heating season are slightly different (Figs. 9A and $9 \mathrm{~B})$. Also during these two seasons the temperature distributions in the ground differ from each other (Figs. 9C and 9D). As a result, the values of the unit heat flux extracted from the ground (Fig. 10) and of the heat pump thermal performance (Fig. 11) are a bit different in season I and II. Cooling the building in summer does not have much effect on the initial temperature distribution in the ground (Fig. 9E), i.e., before the beginning of the second heating season (season IIA). Therefore, there are only slight differences between the unit heat fluxes extracted from the ground in seasons II and IIA (Fig. 10) as well as the heat given up by the VCHP in the heated space in mentioned seasons (Fig. 11). The temperature of the intermediate agent results from the cooperation of the VCHP with the subsystem ground - GHE and varies throughout the heating season. The changes in this temperature in the cases under consideration are presented in Fig. 12. The curves illustrating changes in the intermediate agent mass flow rate (Fig. 13) are similar due to the dependence of its properties on temperature.

As it was also proved by previous analyses $[10,11]$, there are interdependences between the system characteristic parameters. This is the effect of the interrelations between three cycles of the heating water, the refrigerant, and intermediate agent, respectively. Another element that has an impact on the system operation is the environment with changeable parameters.

Due to the cyclic heat extraction in the GHE process of falling tem- 

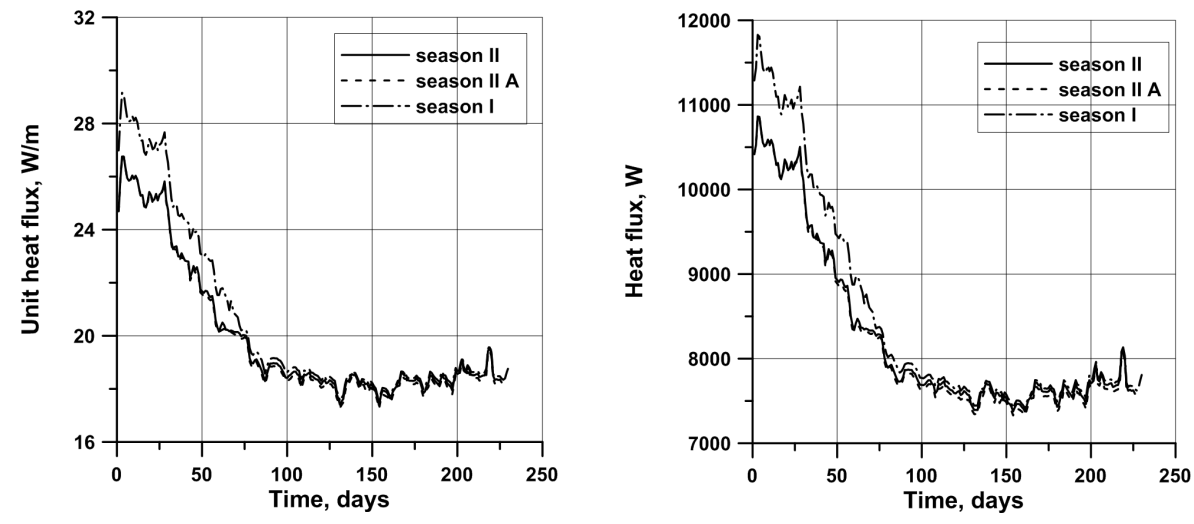

Figure 10: Changes in the average unit Figure 11: Changes in the average heat heat flux transferred from the ground to the GHE per $24 \mathrm{~h}$. flux transferred from the VCHP to the heated space per $24 \mathrm{~h}$.
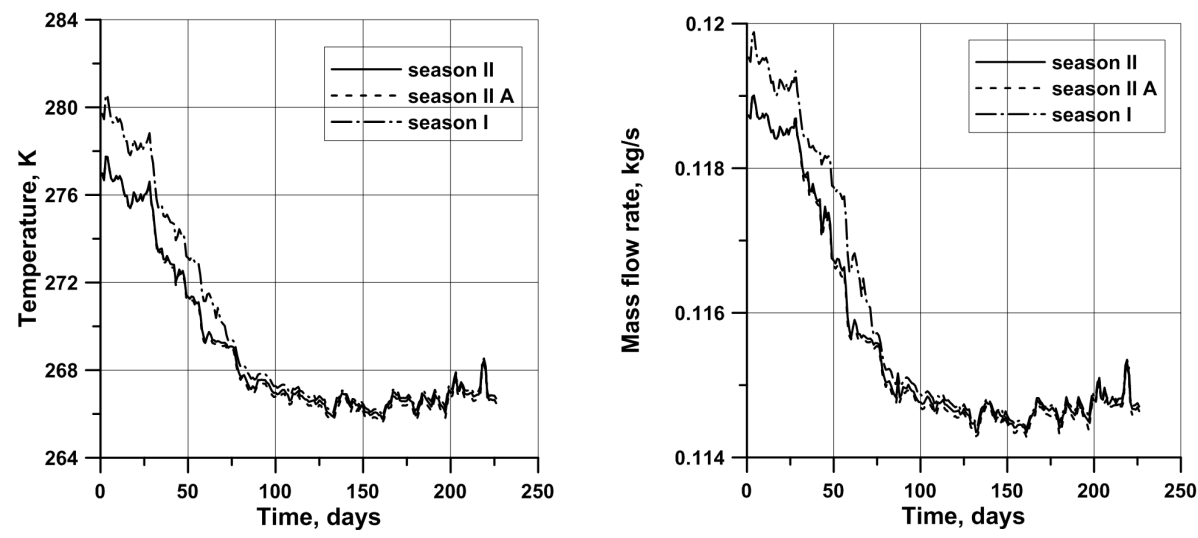

Figure 12: Changes in the average temper- Figure 13: Changes in the average mass ature of the heat carrier in the GHE. flow rate of the heat carrier in the GHE (one tube).

perature of the ground takes place (Figs. 9A-9C). Operation time of the heating system in each balance period is dependent on the required demand for heat and the thermal capacity of the VCHP. During the down time of VCHP the thermal state of the ground partly recovers. When the heat demand increases, operation time also increases (Fig. 14) while the heat flux collected from the ground and transferred from the VCHP to the heated space decreases (Figs. 10, 11). In the periods of peak demand for 
heat the VCHP operates in a continuous mode and it is supported by the additional heat source. Increase of the unit heat fluxes collected in the GHE and values of the thermal capacity of the VCHP within the end of the heating seasons result from the decrease of the heat demand (Figs. 10 and 11). At that early spring period the operation times in balance periods decrease and processes of natural thermal regeneration in the ground are more intense. Changes in the temperature of the heat carrier in the GHE and its mass flow rate, presented in Figs. 12 and 13 are related to thermal state of the ground in the vicinity of the tubes.

In the two cases under consideration, the curves illustrating the time of operation per 24 hours (Fig. 14) are very similar and the building cooling process has a very slight impact on the change in the use of the additional heat source. The variations in the amount of heat supplied from this source in season IIA are shown in Fig. 15.

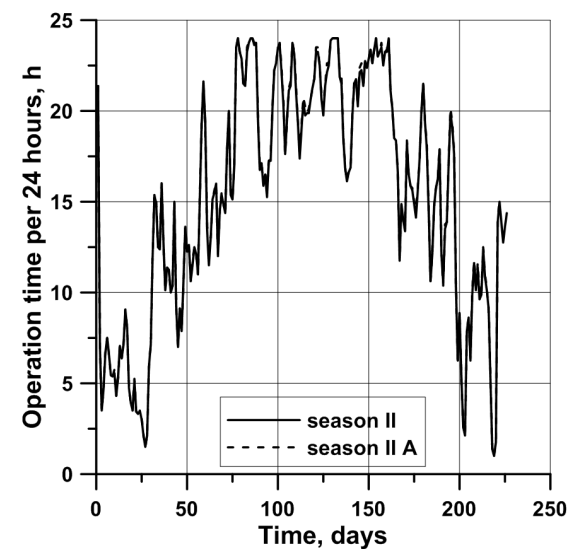

Figure 14: Operation time of the VCHP compressor per 24 hours.

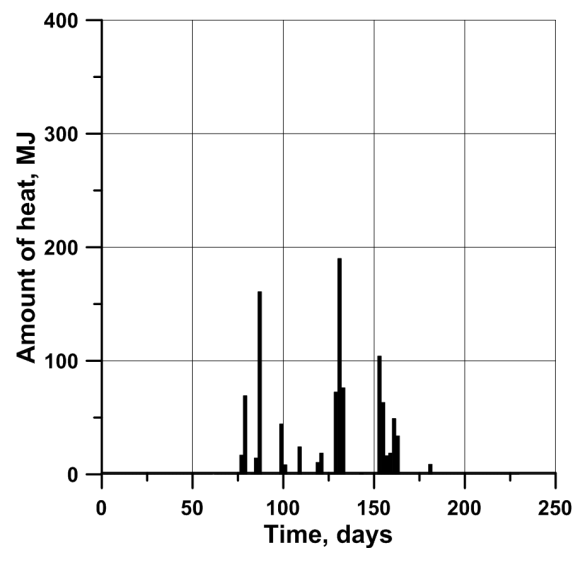

Figure 15: Changes in the amount of heat transferred from the additional heat source to the heated space (season IIA).

\subsection{Calculation results of the cooling system}

In the case of the building cooling, the temperature distribution in the rock mass at the beginning of summer (Fig. 16A) was determined as the effect of thermal calculations taking account of the natural thermal regeneration of the ground area around the GHE tubes between the end of the first heating season and the moment that the cooling system started to operate. One 
K

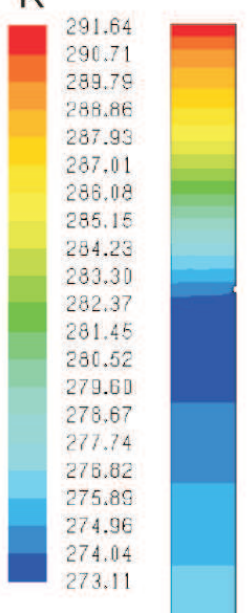

B

$\mathrm{K}$

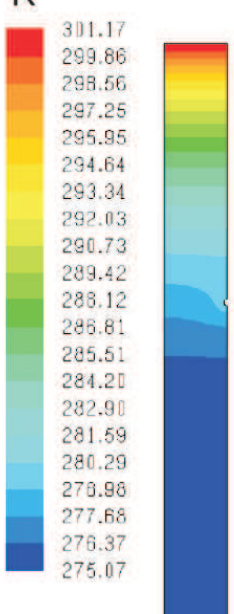

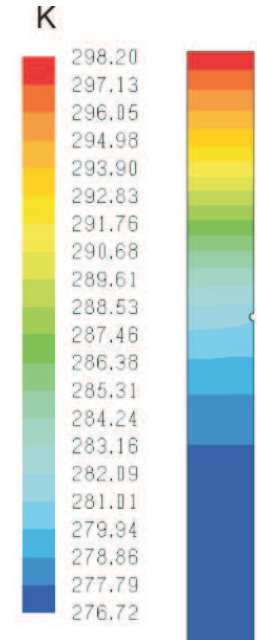

Figure 16: Temperature distributions in the ground: A) beginning of summer (278th day), B) 289th day, C) 302nd day.

of the calculated quantities is the time-dependent heat flux extracted from air in the building (Fig. 17). The heat flux values decrease because the temperature distribution in the ground around the tubes of GHE during the cooling system operation changes - the temperature rises and from a certain moment it is no longer possible to cool the building. The building cooling process has a cyclic character. The time of operation per $24 \mathrm{~h}$ is presented in Fig. 18. In the first case, the cyclic results from the break in the system operation after the required amount of heat is extracted from the building in a given balance period. In the second case, the cyclicity is related to the fact that no more heat can be given up to the ground due to the temperature rise around the GHE tubes. As it was already mentioned, in such cases a break in the cooling system operation is necessary before heat can be transferred into the ground again. The analyzed cooling system operates for the first 10 days (i.e., from the 278th to the 288th day of the season, counting from the beginning of the first heating season). On the 11th day it operates only for several hours, further cooling is impossible. During the break in the system operation, ground temperature drop occurs in the vicinity of the GHE tubes (Fig. 16B) and on the 292nd day the building cooling is again possible for a short time. After more breaks lasting 

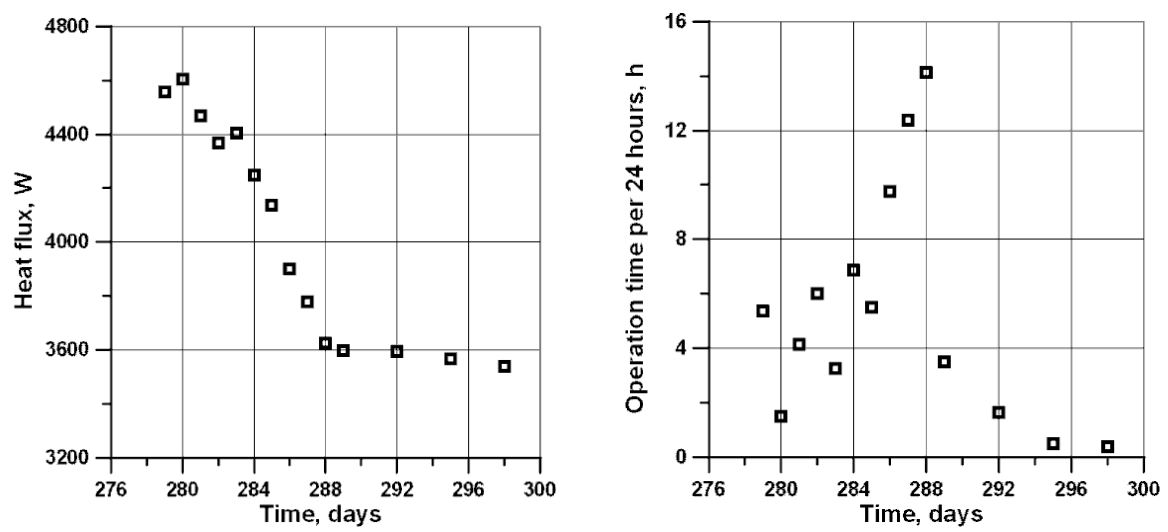

Figure 17: Changes in the average heat Figure 18: Operation time of the cooling flux transferred from the cooled space to the ground per $24 \mathrm{~h}$. system per $24 \mathrm{~h}$.

48 and 72 hours, the system switched on twice but operated for a very short time only. The rise in the ground temperature around the GHE tubes that can be observed in Fig. 16C on the 302nd day results from the impact of the environment. From that moment on, the building cooling is no longer possible because the ground temperature around the tubes continues to rise and - until the beginning of the second heating season - the ground natural thermal regeneration takes place (Fig. 9E). The changes in temperature and in the mass flow rate of the intermediate agent shown in Figs. 19 and 20, respectively, correspond to the changes in the heat flux transferred to the ground (Fig. 17). The cooling system made a more or less $17 \%$ contribution to satisfy the total demand for cool in the period under consideration and the system operation time was $75 \mathrm{~h}$.

\subsection{Energy consumption}

The results of the thermodynamic calculations were used to determine the cumulative energy consumption and harmful emissions caused by the generation of electricity needed to produce heat and cool. The calculations were performed at the power plant efficiency of 0.36 , electricity conversion and transmission efficiency of 0.92 , cumulative energy consumption for hard coal of $1.064 \mathrm{MJ} / \mathrm{MJ}$ [15] and coal calorific value of $20.18 \mathrm{MJ} / \mathrm{kg}$. The total consumption of electricity in the heating system is presented in Tab. 1. It was assumed that the additional heat source was an electrical 

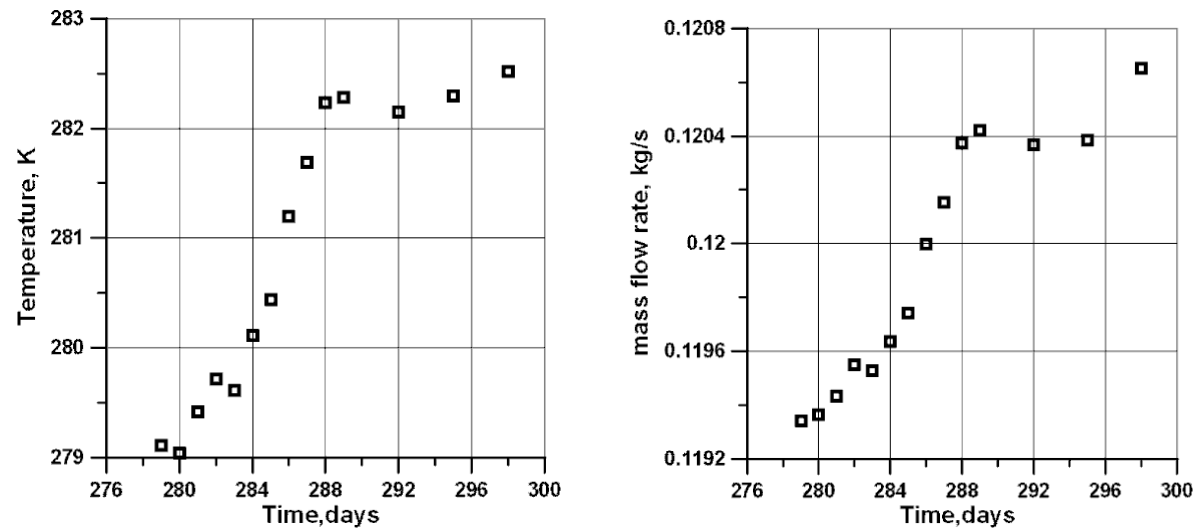

Figure 19: Changes in the average tem- Figure 20: Changes in the average mass perature of the intermediate medium in the GHE during the flow rate of the heat carrier in building cooling per $24 \mathrm{~h}$. the GHE during the building cooling per $24 \mathrm{~h}$ (one tube).

device (with assumed efficiency equal to 1), and the consumption of energy by water pumps was ignored. Due to the incomplete thermal regeneration of the ground in the spring-summer period, the cumulative consumption of chemical energy is smaller in season I compared to season II (Tab. 1). This concerns both analyzed variants. Generating the amount of heat required in the system under analysis in a coal-fired heating station (station efficiency - 0.88, transmission efficiency - 0.95) involves a higher consumption of fuel, by about $480 \mathrm{~kg}$ compared to the system with the VCHP (season I). The calculation results indicate that cumulative energy consumption is lower if the cooling system uses a GHE (season IIA) in comparison with season II, but the difference is slight. Additional, beneficial effect is the partial cooling of building, using GHE.

Table 1: Energy consumption of the heating system during heating seasons.

\begin{tabular}{|l|r|r|r|}
\hline & Season I & Season II & Season IIA \\
\hline \hline Electric energy, MJ & 33708 & 33957 & 33896 \\
\hline $\begin{array}{l}\text { Energy from the addi- } \\
\text { tional heat source, MJ }\end{array}$ & 2094 & 2370 & 2367 \\
\hline $\begin{array}{l}\text { Cumulative energy con- } \\
\text { sumption in the heating } \\
\text { system, MJ }\end{array}$ & 115016 & 116702 & 116497 \\
\hline
\end{tabular}


The comparative analysis was also conducted assuming that the amount of heat taken by the cooling system comprising the GHE could be extracted from the building using a compressor-based cooling device with a performance coefficient of 2.5. Cumulated primary energy consumption needed to drive the compressor is equal to $1390 \mathrm{MJ}$, whereas in the cooling system with GHE this value is equal to $164 \mathrm{MJ}$. It should be emphasized that these values were calculated for assumed parameters of the cooling system, the GHE and the building. As mentioned before the effect of cooling system operation was depended on thermal state of the ground and the same on the parameters of the heated system: heated building - VCHP - GHE.

In Poland, where electricity is generated mainly in coal-fired power plants, the operation of the analyzed systems is indirectly burdened with harmful emissions to the environment. Cumulative hard coal consumption and harmful emissions connected with building heating (season II and IIA) and partial building cooling are presented in Tab. 2.

Table 2: Cumulative coal consumption and harmful emissions.

\begin{tabular}{|c||c|c|c|c|c|c|}
\hline Season & Coal, $\mathrm{kg}$ & $\mathrm{CO}_{2}, \mathrm{~kg}$ & $\mathrm{SO}_{2}, \mathrm{~kg}$ & $\mathrm{NO}_{x}, \mathrm{~kg}$ & $\mathrm{CO}, \mathrm{kg}$ & Ash, $\mathrm{kg}$ \\
\hline \hline II & 5851 & 11390 & 17.3 & 19.9 & 10.8 & 2.17 \\
\hline IIA & 5780 & 11252 & 17.1 & 19.7 & 10.7 & 2.14 \\
\hline
\end{tabular}

\section{Final comments and conclusions}

The presented results of thermodynamic calculations, as the previous analyses $[10-12]$, prove that the parameters of the heating system operation, the heated building, and the subsystem: ground - horizontal ground heat exchanger are correlated. As mentioned above, the parameters are the effect of a combination of three systems of the medium circulation. The additional element that affects the demand for heat (cool), as well as the thermal processes in the ground and the ground heat exchanger operation, is the environment. The parameters characterizing the operation of the heating and cooling system under consideration vary in time [10-12]. In the analyzed case of cooling the building using the GHE, the impact of a rise in the ambient temperature on the temperature distribution in the ground in the vicinity of the ground heat exchanger tubes is clearly visible. And this also affects the possibility of heat extraction from the building. 
The possibility also depends on the ground thermal state after the first heating season. As shown the results of earlier analyses concerning heating systems with VCHP and GHE [10,12], the thermal state of the ground is related, among other thinks, to the ground heat exchanger geometrical parameters. The advantages gained from the proposed cooling and heating system in terms of energy and ecology could be bigger or smaller depending on depth of the location of tubes or the spacing between them. The computer code enables multivariate computations in order to select a most favourable configuration of GHE on account of total energy consumption in heating and cooling system.

A very important factor that impacts on the heating and cooling system operation is a building structure. It has been shown the variability of heat demand has an influence on the thermal state of the ground and on the possibility of buildings cooling in summer. These analyses also demonstrate the impact of the utilization of the cooling system on the functioning of the heating system in the next heating season. As mentioned before, the mutual connections take place between individual elements of the heating (cooling) system. Due to this fact and due to the correlations between operation of the heating and cooling system possibility of ground utilization and energy consumption are dependent on technical parameters of these systems, their functioning modes and a building structure and location.

The cumulative method of energy consumption calculation shows the real effect on the depletion of fuel resources. Presented results - cumulated primary energy consumptions - are characteristic only for specific building structure, parameters of the heating and cooling systems and theirs operation modes. Environmental impact of similar systems results from factors mentioned above but mainly from the electric power system structure.

Acknowledgement This work was funded by the Ministry of Science and Higher Education: partly under research project NN 512317238 from science financial resources 2010-2012 and partly under Statutory Research Funds of the Faculty of Energy and Environmental Engineering, Silesian University of Technology (Poland).

Received 23 June 2015

\section{References}

[1] ANSYS Fluent, http://www.ansys.com 
[2] Demir H., Ahmet Koyun A., Temir G.: Heat transfer of horizontal parallel pipe ground heat exchanger and experimental verification. Appl. Therm. Eng. $\mathbf{2 9}(2009), 224-233$.

[3] Esen H., Inalli M., Esen M.: Numerical and experimental analysis of a horizontal ground-coupled heat pump system. Build. Environ. 42(2007), 1126-1134.

[4] Yang H., Cui P. Fang Z.: Vertical-borehole ground-coupled heat pumps: A review of models and systems. Appl. Energ. 87(2010), 16-27.

[5] Cui P., Yang H., Spitler J.D., FAng Z.: Simulation of hybrid ground-coupled heat pump with domestic hot water heating systems using HVACSIM+. Energ. Build. 40(2008), 1731-1736.

[6] Yang H., Cui P. Fang Z.: Vertical-borehole ground-coupled heat pumps: A review of models and systems. Appl. Energ. 87(2010), 16-27.

[7] DąBrowski J., Hutnik E.: Impact of the use of the solar installation and a heat pump in residential building on reduction of conventional energy consumption. Rynek Energii 102(2012), 5, 153-158.

[8] Gupta R., Irving R.: Development and application of a domestic heat pump model for estimating $\mathrm{CO}_{2}$ emissions reductions from domestic space heating, hot water and potential cooling demand in the future. Energ. Build. 60(2013), 60-74.

[9] Sekret R., Nitkiewicz A.: Exergy analysis of performance of low-temperature district heating system with geothermal heat pump. Arch. Thermodyn. 35(2014), 1, $77-86$.

[10] Hanuszkiewicz-DrapaeA M.: Thermal Analysis of Building Heating System with a Vapour Compressor Heat Pump and a Tubular Ground Heat Exchanger. Monography 314, Wydawnictwo Politechniki Śląskiej, Gliwice 2011 (in Polish).

[11] Hanuszkiewicz-Drapala M., Skeadzień J.: Operation characteristics of heat pump system with ground heat exchangers. Heat Transfer Eng. 33(2012), 7, 629641.

[12] Hanuszkiewicz-Drapata M., Skeadzień J.: Heating system with vapour compressor heat pump and vertical U-tube ground heat exchanger. Arch. Thermodyn. 31(2010), 4, 93-110.

[13] SkŁadzień J.: Thermal and Economical Analyses of Ground Freezing. Zeszyty Naukowe Politechniki Śląskiej, ser. Energetyka 78, Gliwice 1981 (in Polish).

[14] Typical Meteorology and Statistical Years. Climatic data for Poland to Thermal Calculations of Buildings. (in Polish) www.mi.gov.pl, (accessed on 2009).

[15] Szargut J., ZięBiK A.: Fundamentals of Thermal Engineering. PWN, Warsaw 1998 (in Polish). 


\section{Appendix}

Fundamental equations describing operation of the heat exchangers in the cooling system

Evaporator:

$$
\begin{gathered}
\dot{Q}_{e}=\dot{m}_{g l} c_{g l}\left(T_{1 g l}-T_{2 g l}\right), \\
\dot{Q}_{e}=\dot{V}_{1 r} \rho_{1 r}\left(h_{1 r}-h_{4 r}\right), \\
\dot{Q}_{e}=k_{e} A_{e} \frac{T_{1 g l}-T_{2 g l}}{\ln \frac{T_{1 g l}-T_{e}}{T_{2 g l}-T_{e}}} .
\end{gathered}
$$

Condenser:

$$
\begin{gathered}
\dot{Q}_{c}=\dot{m}_{w} c_{w}\left(T_{2^{\prime} w}-T_{1 w}\right), \\
\dot{Q}_{c}=\dot{V}_{1 r} \rho_{1 r}\left(h_{2 r}-h_{3 r}\right), \\
\dot{Q}_{c}=k_{c} A_{c} \frac{T_{2^{\prime} w}-T_{1 w}}{\ln \frac{T_{c}-T_{1 w}}{T_{c}-T_{2^{\prime} w}}} .
\end{gathered}
$$

Conventional heat source:

$$
\dot{Q}_{s}=\dot{m}_{w} c_{w}\left(T_{2 w}-T_{2^{\prime} w}\right) .
$$

Heat exchangers in the heated space

$$
\begin{aligned}
\dot{Q}_{h s} & =\dot{m}_{w} c_{w}\left(T_{2 w}-T_{1 w}\right) \\
\dot{Q}_{h s} & =(k A)_{h s e} \frac{T_{2 w}-T_{1 w}}{\ln \frac{T_{2 w}-T_{a}}{T_{1 w}-T_{a}}}
\end{aligned}
$$

Scroll compressor:

$$
\begin{gathered}
N_{e l}=N\left(T_{c}, T_{e}\right), \\
\eta_{i s}=\eta\left(T_{e}, T_{c}\right) .
\end{gathered}
$$

Valve:

$$
h_{3 r}=h_{4 r} .
$$




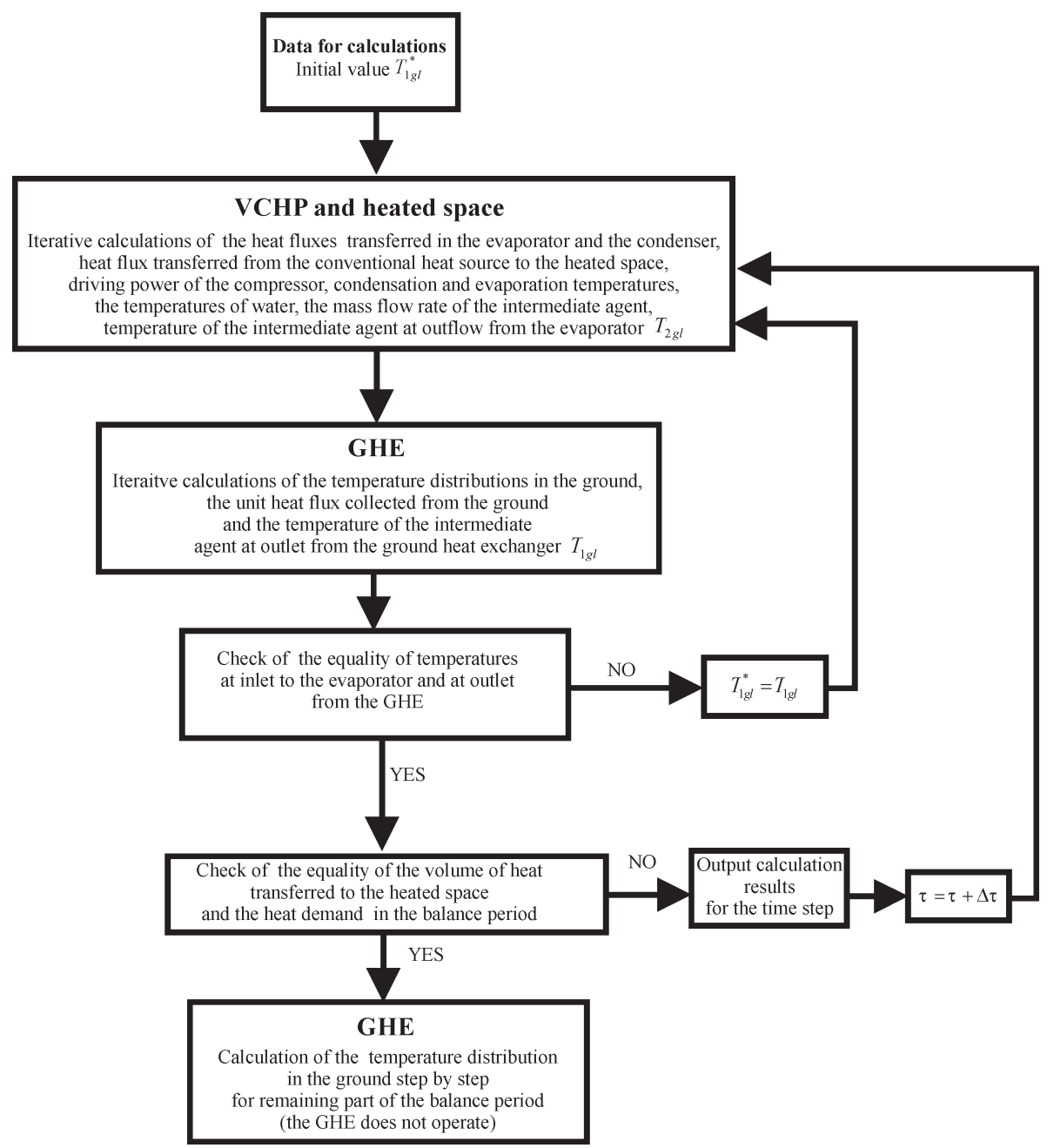

Figure 21: Simplified diagram of the iterative calculations of the heating system with VCHP and GHE.

Fundamental equations describing operation of the heat exchangers in the cooling system

Heat exchangers in the cooling space:

$$
\begin{aligned}
\dot{Q}_{c s} & =\dot{m}_{w} c_{w}\left(T_{4 w}-T_{3 w}\right), \\
\dot{Q}_{c s} & =(k A)_{c s e} \frac{T_{4 w}-T_{3 w}}{\ln \frac{T_{a}-T_{3 w}}{T_{a}-T_{4 w}}} .
\end{aligned}
$$


Additional heat exchanger (water-intermediate agent):

$$
\begin{gathered}
\dot{Q}_{c s}=\dot{m}_{g l} c_{g l}\left(T_{6 g l}-T_{5 g l}\right) \\
\dot{Q}_{c s}=k_{H E} A_{H E} \frac{\left(T_{3 w}-T_{5 g l}\right)-\left(T_{4 w}-T_{6 g l}\right)}{\ln \frac{T_{3 w}-T_{5 g l}}{T_{4 w}-T_{6 g l}}} .
\end{gathered}
$$

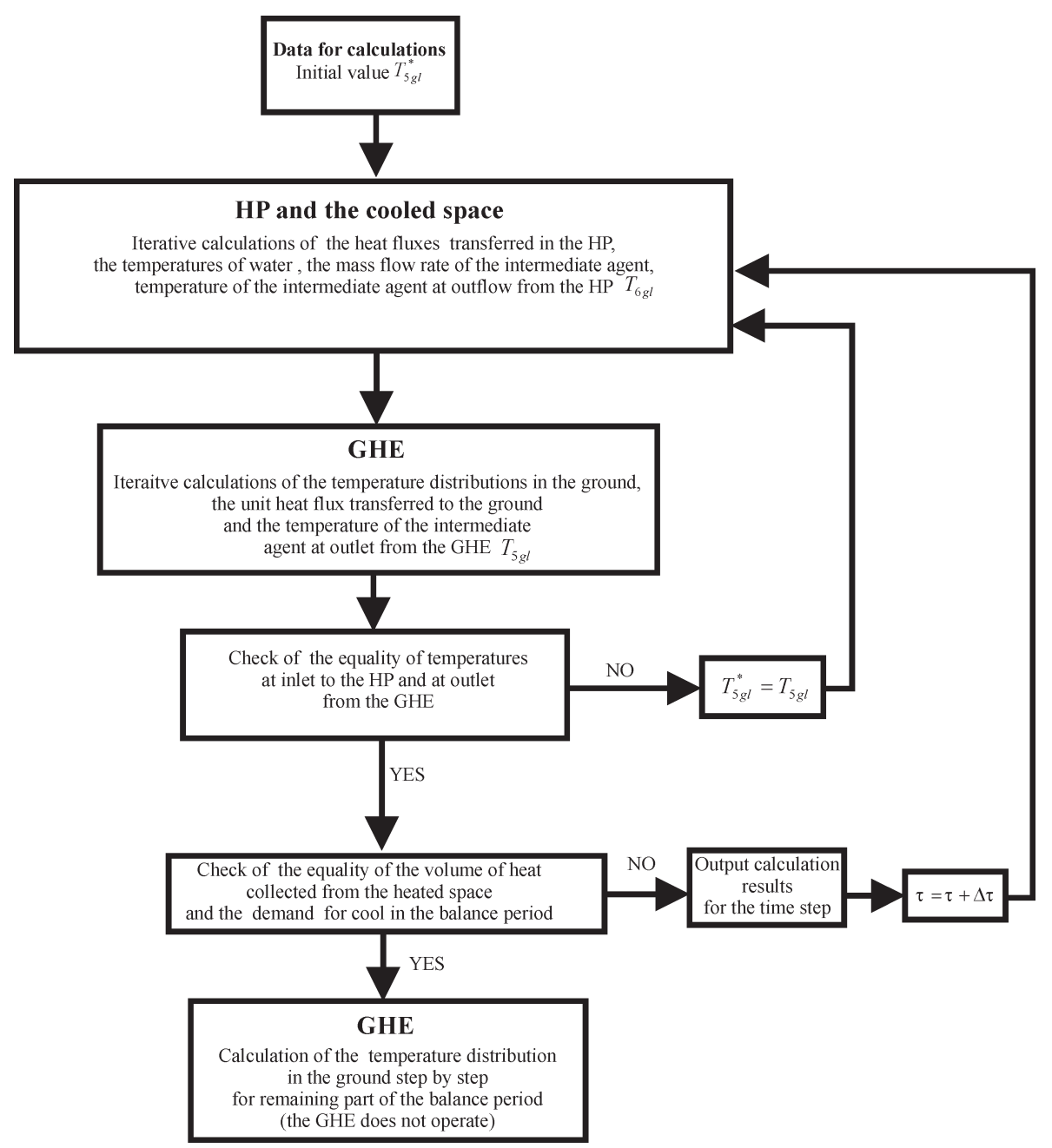

Figure 22: Simplified diagram of the iterative calculations of the cooling system. 